\title{
Utilisation des bioessais " in situ " (substrats artificiels) pour caractériser la qualité des eaux de rivière à l'aide du périphyton
}

\author{
T. Watanabe 1 \\ J. Capblancq2 \\ A. Dauta ${ }^{2}$
}

Mots clés : Bioessais, périphyton, qualité des eaux courantes, substrats artificiels.

La dynamique de croissance du périphyton sur des substrats artificiels immergés à l'amont et à l'aval de secteurs de cours d'eau recevant des rejets polluants a été utilisée pour évaluer le niveau de pollution. La méthode est basée sur la détermination de paramètres globaux (matières organiques, pigments chlorophylliens, respiration, photosynthèse, assimilation hétérotrophe) qui permettent de caractériser le développement des communautes photoautotrophes et hétérotrophes sur les substrats. Le taux de croissance et l'activité hétérotrophe de ces communautés évoluent au cours de la durée d'immersion des substrats selon un modè le géné ral qui est décrit à partir de séries de données recueillies sur trois cours d'eau. Les résultats obtenus sur 22 stations montrent que ces paramètres constituent des indicateurs de pollution plus sensibles que ceux basés sur la cumposition spécifique des communautés de périphyton.

Artificial substrata as an a in situ $n$ bioassay for assessing water quality in rivers by means of periphyton.

Keywords : Bioassays, periphyton, Ereshwater quality, artificial substrates.

The dynamics of periphyton growth on artificial substrata submerged in upstream and downstream stations of stretches of rivers receiving pollution loads has been used to evaluate levels of pollution. The method is based on the determination of global parameters (organic matter, photosynthetic pigments, respiration, photosynthesis, heterotrophic assimilation) characterizing the development of photoautotrophic and heterotrophic communities on artificial substrates. Changes in growth rate and metabolic activity during the immersion period of the substrata follow a general pattern described from serics of data collected in three rivers. The results provided by artificial substrata in 22 stations show that these simple parameters are more sensitive indicators of water pollution than indices based on specific composition of algal communities.

\section{Introduction}

Le périphyton sensu lato i.e l'ensemble de tous les organismes vivant à la surface des objets immergés dars l'eau (WETzEL 1983) uccupe une place inpurtante dans les méthodes de détermination de la qualité des eaux. Ces methodes se regroupent globalement autour de deux types d'approche:

1. Universidade Federal da Paraiba, CCEN, Campus Universitario I, 58000, Joao Pessoa, PB, Brasil.

2. Laboratoire d'Hydrobiologie, UA CNRS 695, Université Paul Sabatier 118, route de Narbonne 31062 Toulouse Cédex France.
1) celles qui font appel aux notions d'organismes indicateurs et de diversité spécifique des communautés (Descy 1976-1979; Coste 1978; LangeBerthalot 1979; Sullivan 1986) et nécessitent une connaissance approfondic de la taxonomic d'un ou plusieurs groupes,

2) celles qui considèrent que le taux de croissance du périphyton représente, indépendamment des espèces qui le composent, une réponse directe aux conditions physiques et chimiques du milieu (Wührman 1974; Eichenberger 1975; Wührmann \& Eichenberger 1975 ; Capblancq \& Cassan 1979a, b ; Sand-Jensen 1983). L'utilisation de systèmes 
artificiels (canaux expérimentaux, subst rats artificiels) permettant d'uniformiser les conditions de croissance vis-à-vis des facteurs physiques (lumière, courant), il est alors théoriquement possible d'inter. préter les variations du taux de croissance en fonction de la qualité chimique de l'eau.

Partant des résultats obtenus par Capblancq \& Cassan (1979a,b) lors de l'étude d'une rivière fortement polluée, cette seconde approche a été développée dans les buts

- d'analyser la dynamique de croissance du périphyton sur les substrats artificiels immergés dans des secteurs de cours d'eau recevant des charges différentes d'éléments nutritifs, minéraux et organiques,

- d'évaluer les possibilités d'application de cette méthode pour caractériser l'état de pollution des eaux.

\section{Matériel et méthodes}

\subsection{Stations d'étude}

Les expériences destinées à définir la dynamique de colonisation des substrats artificiels par le périphyton furent conduites au printemps (mai-juin 1983) et en automne (octobre-novembre 1983) sur des points situés à l'amont et à l'aval de rejets de stations d'épuration d'effluents domestiques dans trois ruisseaux de la banlieue toulousaine. Les relevés des substrats artificiels immergés dans chaque station au début de la période d'étude ont été échelonnés sur une periude de 4 à 5 semaines. Un ensemble de 22 stations a été choisi sur le réseau hydrographique du bassin garonnais ${ }^{1}$ pour tester les possibilités d'application de ce bioessai. Ces stations (/ig. 1 et Tableau I) se caractérisent par des rejets de pollutions diverses, d'origines domestique ou industrielle. L'étude a été conduite en juin-juillet 1984.

\subsection{Variables mesurées}

Chaque station est caractérisée par un ensemble de données concernant :

1. Nous tenons à remercier M. Roux et l'Agence Financière de Bassin Adour-Garonne pour la collaboration apporté dans ce travail.

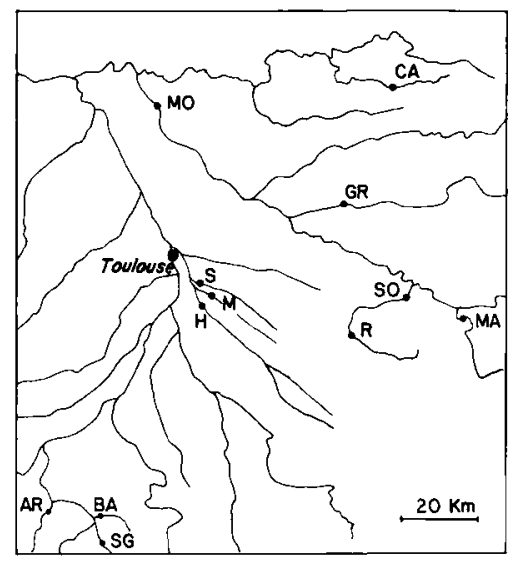

Fig. 1 : Localisation des stations d'échantillonnage.

Tableau I : Types de rejets sur les stations étudices. (signification des indices. ex. : $\$ 1$ identifie le point de rélé. rence en amont du rejet, $S 2$ le point en aval).

\begin{tabular}{|l|l|}
\hline Identification & \multicolumn{1}{|c|}{ Type de rejet } \\
\hline S & Station d'épuration \\
M & Station d'épuration \\
R & Stationd'épuration \\
So & Abattoir \\
MA & Industrie Chimique \\
GR & Industrie Lainière \\
CA & Megisserie \\
MO & Industrie Chimique \\
SG & Papeterie \\
BA & Laiterie \\
AR & Laiterie \\
\hline
\end{tabular}

- la physicochimie de l'eau, définie à partir d'analyses d'échantillons prélevés au début et à la fin de la période d'immersion des substrats artificiels

- les propriétés du périphyton développé sur les substrats artificiels ; ces derniers sont constitués de feuilles de polyéthylène fixées sur des supports en PVC de $8 \mathrm{~cm}$ de diamètre et $20 \mathrm{~cm}$ de hauteur (fig. 2).

Les feuilles de polyéthylène colonisées ramenées au laboratoire dans un container complété avec l'eau 


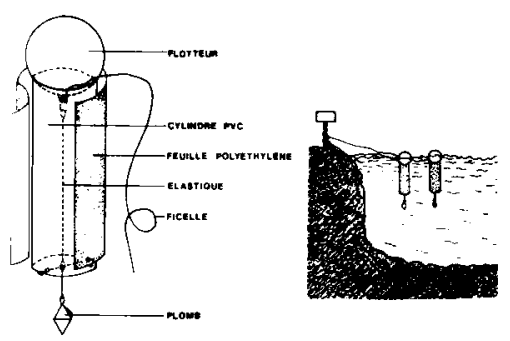

Fig. 2 : Schéma du substrat artiliciel. Substrat artificiel en place dans le cours d'eau.

du milieu, sont immédiatement divisées en fraction homogènes afin de procéder aux expérimentations et aux mesures. Le tableau II résume l'ensemble des paramètres tirés des mesures réalisées sur les biodermes. Les techniques utilisées sont décrites de façon plus détaillees par Capblancq \& Cassan (1979a) et Watanabe (1985).

\section{Résultats}

\subsection{Dynamique de croissance du périphyton sur les substrats artificiels}

Les figures 3 à 6 illustrent, à titre d'exemple, l'évolution des variables mesurées au cours de la période printanière dans les stations $\mathrm{M} 1$ (amont du rejet) et M2 (aval du rejet) d'un cours d'eau recevant des aux provenant d'une station d'épuration.

\section{PROPRIETEES CHIMIQUES}

L'influence du rejet se manifeste par des effets visibles sur le $\mathrm{pH}$, les M.E.S. et les substances nutritives ( $f$ ig. 3 ). Les différences les plus marquées entre les points amont et aval concernent les concentrations de phosphore, la DBO et les formes de l'azote (forte augmentation de $\mathrm{NH} 4$ et $\mathrm{NO} 2-$, diminution de NO3 - par effet de dilution et par dénitrification).

\section{PARAMETRES DE BIOMASSE}

L'évolution du matériel fixé sur les substrats arti. ficiels en fonction de leur durée d'immersion est décrite dans les figures $4 a$ et $4 \mathrm{~b}$. La biomasse des algues (fig. 4b) est déduite des dosages de chlorophylle (fig. 4c) en considérant que le rapport moyen matières organiques/chlorophylle est égal à 60 (fig. 5) ; la biomasse d'hérérotrophes est ensuite estimée par différence entre le poids total de matières organiques et le poids de matières organiques des algues. Les résultats révèlent les points suivants :

1) La masse de matières organiques représente une faible proportion, de l'ordre de $25 \%$ en moyenne, du poids sec de matériel fixé sur les substrats artificiels. Les quantités élevées de matériel minéral peuvent étre attribućes au piégeage de matières en suspension dans le réseau de filaments et de fibrilles de polysaccharides qui se développent sur les substrats.

2) La colonisation initiale des substrats est due aux communautés hétérotrophes qui représentent plus de $75 \%$ du poids de matières organiques à l'issuc de la premiere semaine d'immersion ; leur biomasse atteint un maximum après 10 à 15 jours d'immersion des substrats.

3) Le développement des algues est plus lent : leur biomasse augmente progressivement au cours de la période d'immersion selon une courbe de type sigmoïde caractéristique d'une succession de phases de colonisation, de croissance et d'équilibre. Cette dernière est atteinte après 4 à 5 semaines d'immersion des substrats.

4) L'influence des rejets sur l'évolution des communau tés photoautotrophes $(\mathbf{P})$ et hétérotrophes (H) apparait de façon évidente en comparant les stations situées à l'amont et à l'aval. La figure 5 regroupe les résultals obtenus dans trois cours d'eau voisins recevant des effluents du mème type. Dans les stations situées à l'amont des rejets, il existe une bonne corrélation $(r=0,968)$ entre la quantité de matière organique fixée sur les substrats et la chlorophylle, indiquant que les algues forment une grande part de la biomasse fixée. Cette relation n'apparaît pas dans les stations situées à l'aval du rejet ou l'on note par contre une forte corrélation $(r=0,895)$ entre le poids de matières organiques et la biomasse d'hétérotrophes.

\section{ACTIVITÉ MÉTABOLIOUE}

L'activité métabolique des communautés varie avec l'âge du périphyton fixé sur les substrats et avec les conditions de milieu (fig. 6). Ainsi : 
Tableuu II : Analyse du bioderme. Paramètres. Techniques, Unités.

\begin{tabular}{|c|c|c|}
\hline PARAMETRES & TECHNIQUES & UNITES \\
\hline $\begin{array}{l}\text { Poids sec } \\
\text { Poids de cendres } \\
\text { latière Organique }\end{array}$ & $\begin{array}{l}\text { Raclage d'une surface } \\
\text { déterminée de substrat }\end{array}$ & $\mathrm{mg} / \mathrm{dm} 2$ \\
\hline $\begin{array}{l}\text { Photosynthèse } \\
\text { Production } \\
\text { primaire }\end{array}$ & $\begin{array}{l}\text { Incubation d'un fragment de } \\
\text { bioderme en présence de } \\
1 \text { HCoßNaH, à lumière saturante } \\
\text { pendant } 2 \text { heures. }\end{array}$ & $\begin{array}{l}\text { mg } c / m g C h l, a / h \\
\text { oul } \\
\text { ag } C / d m 2 / h\end{array}$ \\
\hline Respiration & $\begin{array}{l}\text { Mesure de la consommation d'OL } \\
\text { en } 3 \text { heures, à lobscurite. } \\
\text { par un fragment de bioderme. }\end{array}$ & mg $02 / d m 2 / h$ \\
\hline $\begin{array}{l}\text { Chlorophylle et } \\
\text { Phéopigments }\end{array}$ & $\begin{array}{l}\text { Extraction par éthanol } \\
\text { boull lant sur une surface } \\
\text { déterminée de substrat. }\end{array}$ & He $\operatorname{ch} 1, a / d \ln 2$ \\
\hline $\begin{array}{l}\text { Assimilation et } \\
\text { Dégradation de } \\
\text { glucose }\end{array}$ & $\begin{array}{l}\text { Mesure avec du D.Glucose } 14 \mathrm{C} \\
\text { et capture du } 1 \pm \mathrm{CO} 2 \text { rejeté } \\
\text { par de la Hyamine }\end{array}$ & $\begin{array}{l}\text { Hg glucose } \\
\text { mg Mat. Orga. } \\
\text { heure }\end{array}$ \\
\hline $\begin{array}{l}\text { Identification } \\
\text { des organismes } \\
\text { et comptage }\end{array}$ & $\begin{array}{l}\text { Observation directe du sub- } \\
\text { strat aul microscope, } \\
\text { Preparation des Diatomees. } \\
\text { Comptage par méthode UTERMOHL } \\
\text { apres dilacération, homogé- } \\
\text { néisation. }\end{array}$ & Nbre $c / d \mathrm{~m}^{2}$ \\
\hline
\end{tabular}

- le taux de photosynthèse spécifique passe par un maximum après 8 à 10 jours d'immersion et décroît ensuite très rapidement pour des biodermes plus âgés. L'elfet des rejets polluants sur la photosynthèse apparait surtout pendant les deux premières semaines et se traduit par une forte diminution de l'activité par unité de chlorophylle à l'aval du rejet polluant.

- les courbes d'évolution de la respiration montrent que la consommation d'oxygène par unité de matière organique est plus intense pendant les deux premières semaines et décroit avec l'âge du bioderme. En liaison avec un développement plus important des hétérotrophes, l'influence des rejets se traduit par une forte augmentation de la consommation d'oxygène dans la station aval.

Les travaux réalisés sur le bactérioplancton (Hobbie \& Wright 1965, Herbland \& Bois 1974 par exemple) et sur le periphyton (Capblancq \& Cassan 1979b) montrent que les cinétiques d'assimilation de molécules organiques obéissent à une loi de réaction enzymatique. Celle-ci est décrite par l'équation de Michaelis-Menten dont les paramètres (Vmax, kS) permettent de caractériser le potentiel hétérotrophe d'une communauté de microorganismes ; les résultats de mesures réalisées avec des solutions de glucose marqué au $14 \mathrm{C}$ à des concentrations comprises entre 3 et $25 \mathrm{mg} / \mathrm{l}$ sont représentés dans la figure 7. Les paramètres ds cinétiques évoluent avec l'âge du bioderme selon un schéma identique à l'évolution de l'activité photosynthétique et respiratoire. Le taux maximal d'assimilation du glucose (Vmax) passe par un maximum correspondant à des communautés âgées de 10 à 15 jours ; il décroit ensuite avec l'âge du bioderme, l'augmentation de la couche de périphyton et de biomasse non active diminuant l'activité par unité de matière organique. Cette couche freine également la diffusion du glucose : ceci se traduit par une augmentation du paramètre $\mathrm{kS}$ qui représente par définition la concentration du substrat entrainant la demi-saturation du système enzymatique. 


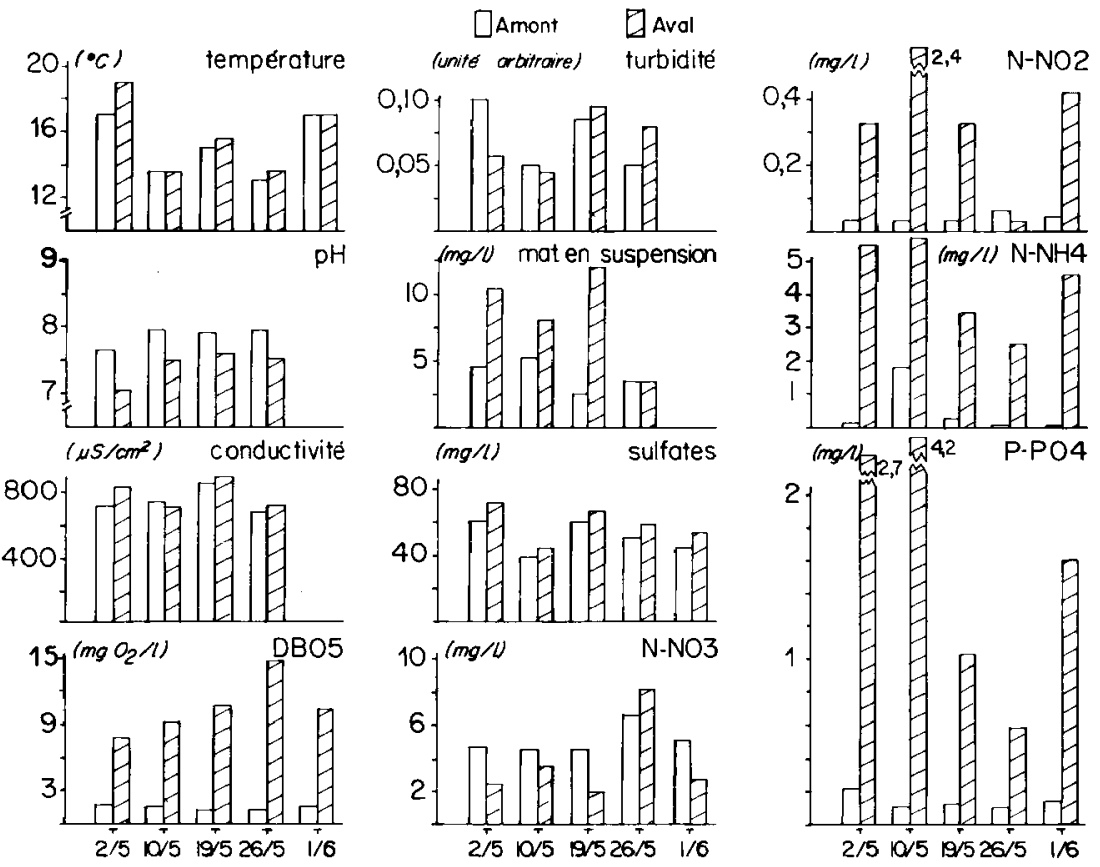

Fig. 3 : Variation des caractéristiques physicochimiques au niveau de la station $\mathbf{M}$, en amont et en aval du point de rejet.

\subsection{Le peuplement d'algues périphériques (fig. 8)}

L'identification et les comptages d'algues nous permettent de suivre la colonisation et la succession des espèces du périphyton sur les substrats artificiels. Une forte corrélation positive $(\mathbf{r}=0,80)$ est observee entre le nombre de cellules et les concentrations en chlorophylle. Cependant elle est plus élevée $(r=0.99)$ lorsqu'il s'agit de substrats jeunes, jusqu'à deux semaines d'immersion. Pour un temps d'immersion prolongé, l'augmentation du nombre de cellules ne se traduit pas par une augmentation correspondante des concentrations de chlorophylle. Cette évolution peut résulter d'une accumulation dans le bioderme de cellules mortes ou sénescentes, d'une diminution de la quantité de chlorophylle par cellule ou d'un changement dela composition spécifique du peuplement.

La communauté périphérique est représentée essentiellement par des Diatomés, des Chlorophy. cées, des Cyanophycées et des Euglénophycées ; les Diatomées forment toujours le groupe le plus important.

Chez les Chlorophycées, les gen res Chlamydomonas et Stigeoclonitum sont les mieux représentés et parmi les Cyanophycées les genres Lyngbya et Pseudanabaena. 

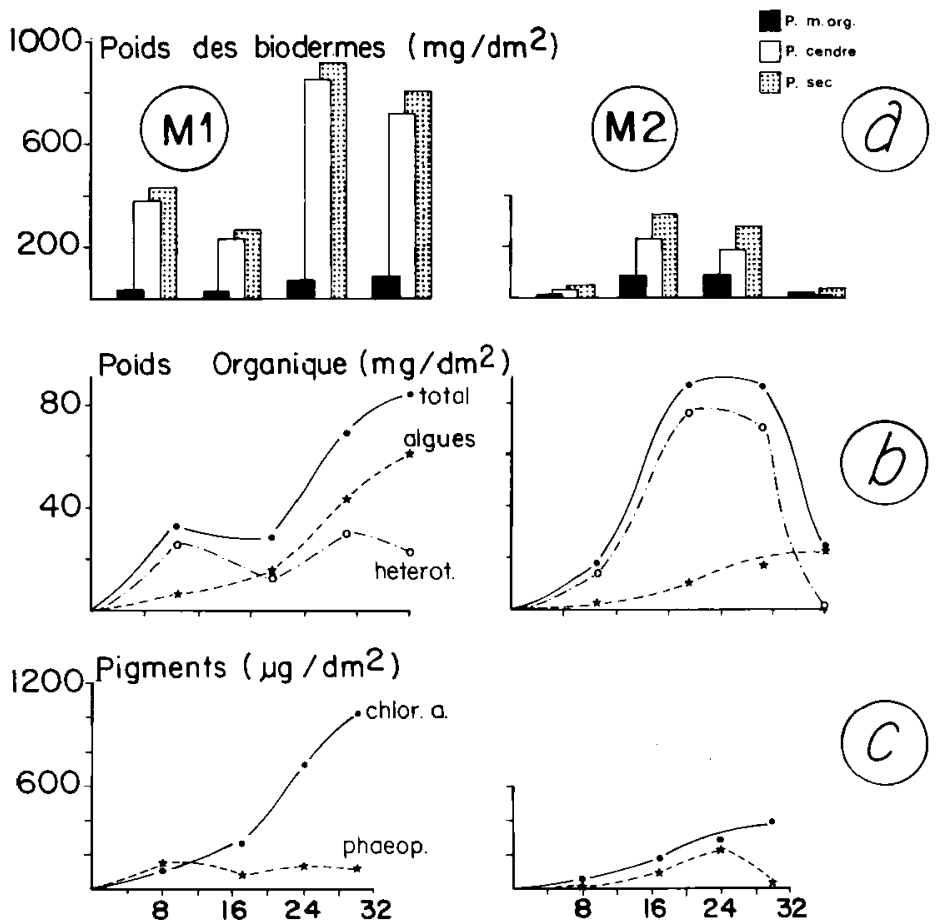

Temps en jours

Fig. 4 : Evolution des paramètres gravimétriques au cours du biotest réalisé à la station $\mathbf{M}, \mathbf{M} 1:$ amont, $\mathbf{M} 2$ : aval.

(a) : poids total de matériel fixé. (b) : répartition autotrophes, hétérotrophes. (c) : pigments chlorophylliens.

Les Diatomées représentent le groupe le plus diversifié avec une très nette dominance des taxas suivants: Achnanthes delicatula ssp hauckiana, Achnanthes lanceolata, Achtantes minutissima, Cocconeis placentula, Cymbella ventricosa, Gomplo. nena parvulum, Navicula cryptocephala v. veneta, Navicula gracilis, Nitzschia dissipata et Surirella ovata. Jes genres Gomphomema, Navictla, Nitzschia et Fragillaria offrent une grande diversité spécifique.

Le nombre d'especes de Diatomés subit une diminution de plus de $40 \%$ entre M1 et M2. La figure 8 représente l'ćvolution de la composition relative des communautés d'algues dans les deux stations. Finalement, après deux semaines, un petit nombre d'espèces (5 à 6) composent $80 \%$ du peuplement. 


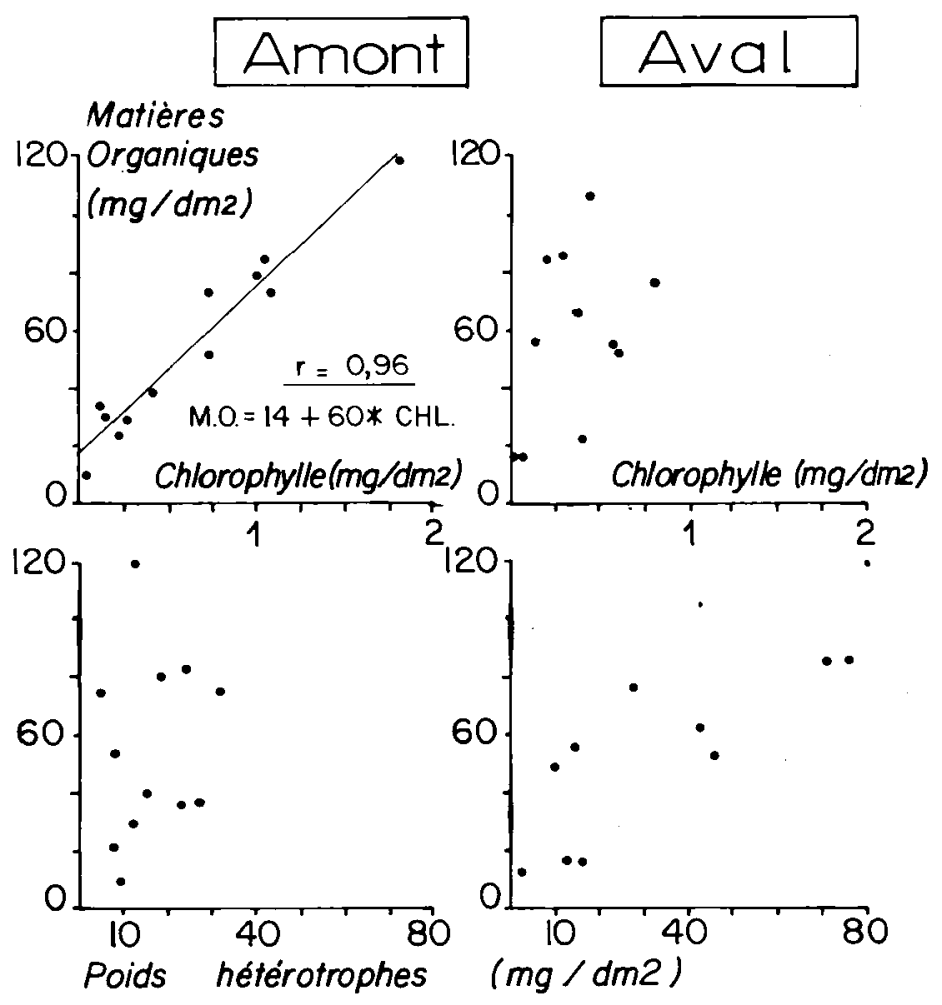

Fig. 5: Rapport entrc la quantité de matières organiques, le poids des algues (exprimé en chlorophylle) et des hétérotrophes, en amont et en aval du rejet, station $\mathbf{M}$.

3.3. Modèle général de développement du périphyton sur substrats artificiels (/ig. 9)

Les données de Capblanca \& Cassan (1979 a, b), relatives à une rivière fortement polluée, et nos résultats concernant les trois cours d'eau montrent que la cinétique de développement du périphyton sur les supports artificiels suit un modile général.
Les paramètres de ce modèle (activité métabolique, taux de croissance et rapport de biomasse des microorganismes autotrophes et hétérotrophes) dépendent des conditions physicochimiques du milieu pendant la phase exponentielle. Au cours de la phase de saturation, la complexité de la communauté augmente et les processus de régulation 

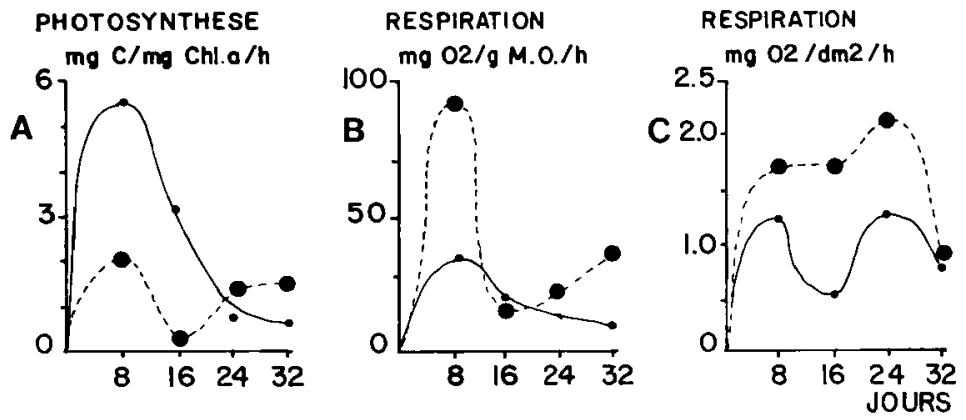

Fig. 6 : Evolution des paramètres liés au métabolisme du périphyton au cours du biotest réalisé à la station $\mathbf{M}$. (a) : photosynthèse, (b) : respiration par unité de biomasse, (c) : respiration par unité de surface.

interne deviennent progressivement plus importants que les échanges avec le milieu aquatique ambiant.

L'interprétation des figures obtenues à partir de l'ensemble des résultats permet d'établir une généralisation sur la dynamique de croissance du périphyton sur les substrats artificiels.

* L'Evolution de la biomasse totale (fig. 9 a) fixée sur les supports, exprimee par rapport à la biomasse maximale (B/Bmax) suit une courbe de type sigmoìde qui atteint sa phase d'équilibre vers la quatrième ou cinquième semaine d'immersion. En considérant séparément l'évolution de la biomasse des hétérotrophes et des autotrophes (fig. 9 b), il faut noter que le modèle d'évolution des autotrophes est identique à celui obtenu pour la biomasse totale. Pour les hétérotrophes, les valeurs maximales sont obtenues entre 15 et 20 jours d'immersion des substrats. Cette évolution met en relief les processus de perte de biomasse par mortalité et décrochage, plus importants chez les hétérotrophes. Elle peut également résulter d'un effet inhibiteur du développement bactérien par les algues.

De fortes similitudes (fig. 9 c) existent égalemenı entre l'évolution de la biomasse totale et
* celle des organismes autotrophes, en amont des rejets,

* celle des organismes hétérotrophes, en aval des rejets.

* Le taux d'accumulation journalier de la biomasse totale $(\mu=\mathrm{d} \mathrm{B} / \mathrm{dt} . \mathrm{B})$ pendant des durées croissantes passc par un maximum entre 10 et 15 jours après l'immersion d'un substrat vierge (fig. 9 d). Cependant, la colonisation des substrats par les hétérotrophes est plus rapide que la colonisation par les algues. Le taux de croissance relatifs $(\mu / 1$ max $)$ des hétérotrophes atteint son maximun entre 8 et 13 jours d'immersion, tandis que les algues présentent des taux plus élevés entre 20 et 25 jours d'immersion. Les valeurs négatives du taux de croissance obtenues pour les organismes hé té rotrophes sont associées aux processus de perte de biomasse, probablement par décrochage et substitution graduelle des algues.

* L'Activité métabolique des biodermes périphytiques, mesurce par la consommation d'oxygene (fig. 9 e), la photosynthèse (fig. 9 f) et l'assimilation hétérotrophe de glucose marqué ( $f \mathrm{~g} .9 \mathrm{~g}$ ) baissent en fonction du temps d'immersion. Ces résul tats montrent que l'activité métabolique du périphyton est 
$V(\mu g \mathrm{glucose} / \mathrm{mq} \mathrm{MO} / \mathrm{h})$
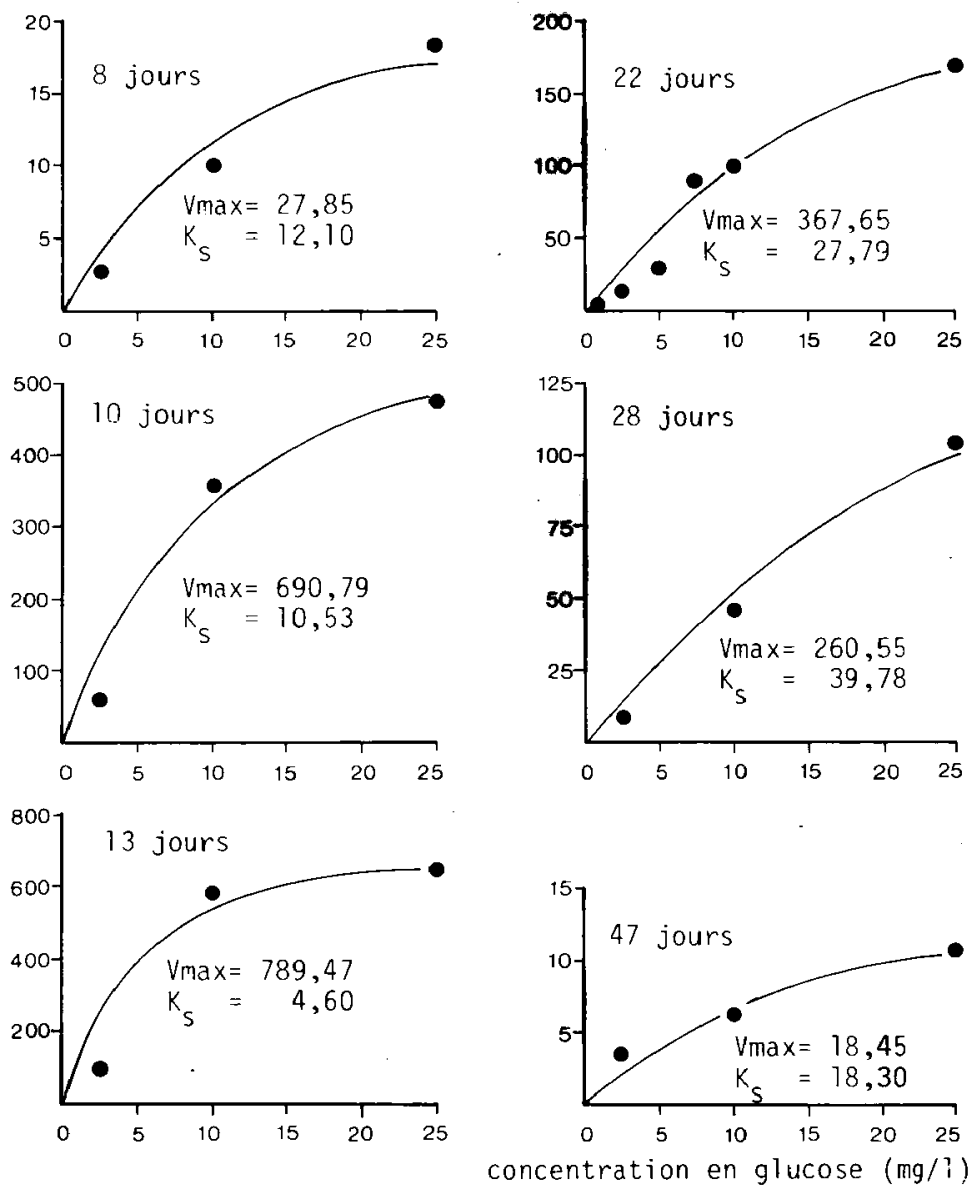

Fig. 7 : Mesure de l'activité hétérotrophe en fonction de l'âge des biodermes. Ajuslement des paramètres selon une cinétique de type Michaelis. 

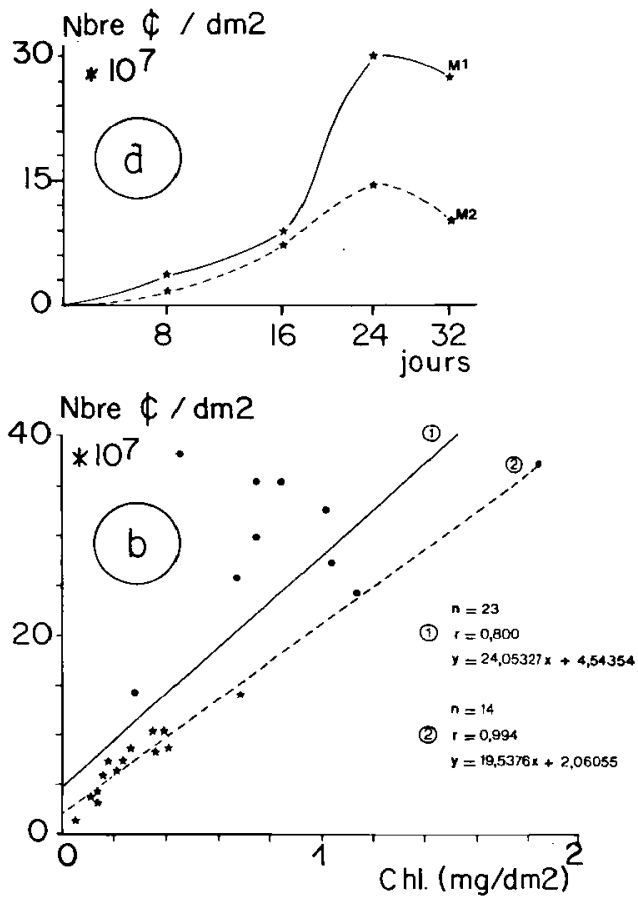
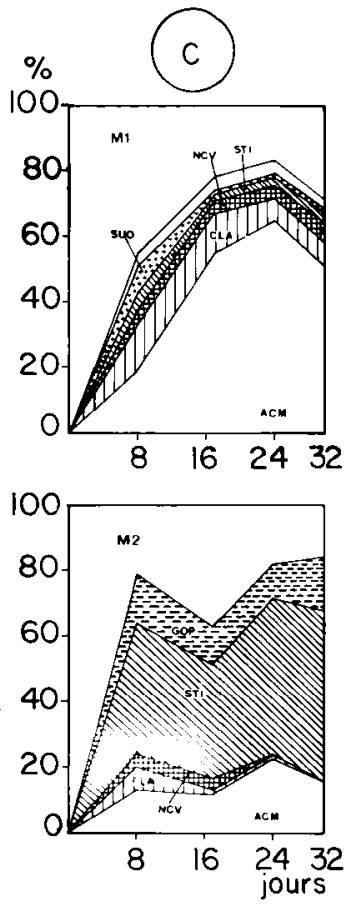

Fig. 8 : Evolution du peuplement d'algues sur les substrats à la station M. (A) Densité des organismes, (B) Relation Chlorophylle/Nombre de Cellules sur l'ensemble des substrats (1) et sur les subst rats immergès depuis moins de 15 jours (2), (C) Abondance relative des espèces (ACM = Achnanthes minutissima. CLA = Chlamydomonas sp., NCV = Navicula cryptocephala var. veneta, GOP = Gomphonema parvulum, SUO = Surirella ovata, STI = Stigeoclonium sp.).

fonction de la densité du bioderme fixé sur les substrats ; celle-ci règle les mécanismes de transport des substances organiques dissoutes et les échanges gazeux au sein de la biomasse.

L'ensemble de ces résultats révèle que les conditions de milieu agissent plus directement sur les parametres de croissance et de metabolisme du bioderme pendant les phases initiales de colonisation des substrats; ce qui est moins évident quand la communauté tend vers un stade climacique. Ainsi, sur le plan méthodologique, les conditions optimales pour les bioessais « in situ " correspondent à des périodes d'immersion des substrats artificiels inférieures à deux semaines.

3.4. Application des bioessais " in situ " à la caractérisation de l'état de pollution des caux courantes

Les possibilités d'utilisation de ce biotest ont été testées en comparant les résultats fournis par la méthode des substrats artificiels dans un ensemble 

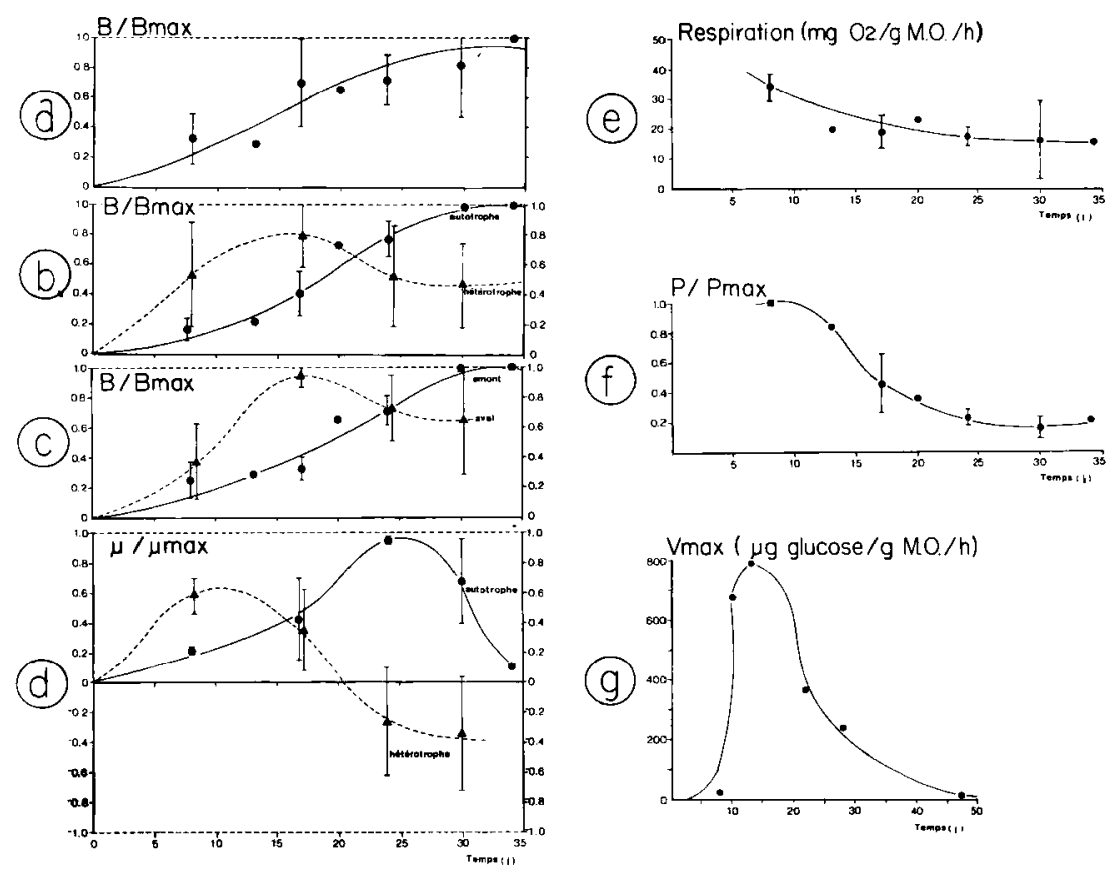

Fig. 9 : Modèle général de développement du periphyton sur les substrats artificiels. L'évolution de la biomasse totale (A), des biomasses d'autotrophes et d'hétérotrophes (B), des biomasses totales à l'amont et à l'aval des rejets (C), du taux d'accumulation journalier de la biomasse (D), de l'activité respiratoire (E), photosynthétique (F) et de l'assimilation hétérotrophe de glucose $(G)$ est décrite à partir de l'ensemble des relevés effectués aux stations $S$, $M$ et $H$ (points $=$ valeurs moyennes; traits verticaux $=$ amplitude).

de 22 stations choisies sur le réseau hydrologique du bassin de la Garonne. Ce's stations se caractéri. sent par des rejets de pollution divers, d'origine domestique ou industrielle (Tableau I).

Dans chacune des stations les substrats ont été immergés pendant 15 jours, durée correspondant à l'ctablissement d'une phase de croissance exponen. tielle du périphyton. Le tableau III résume les donnees physicochimiques mesurées sur ces stations pendant la période de l'étude.
Le traitement des données recueillies sur les 22 stations prospectées a été rćalisé par l'analyse en composantes principales. Près de $70 \%$ de l'inertie totale du système correspond aux quatre premiers facteurs. Les axes 1 et 2 expliquent à eux seuls près de $50 \%$ de la variation (fig. 10 ).

L'analyse de la matrice de corrélation entre les facteurs définis par les quatre premiers axes et les paramètres mesurés, et la représentation dans le 
Tableau III. - Caractérisation des stations étudiées. Données physicochimiques et biologiques. Unités : Conductivité $\left({ }_{1} \mathrm{~S} / \mathrm{cm}\right)$; Température $\left({ }^{\circ} \mathrm{C}\right)$; Alcalinité (mg CaCO3/1); Silice, Sulfate, Nitrates, Matières en suspension, Oxygène dissous, Sodium, Calcium, Magnésium, Potassium et Zinc (mgl) ; Phosphate, Nitrite, Ammoniaque, Pigments chlorophylliens ( $\mu$ g/l); Respiration $\left(\mathrm{mg} \mathrm{02} / \mathrm{dm}^{2} / \mathrm{h}\right)$; Photosynthèse $\left(\mathrm{mg} \mathrm{C} / \mathrm{dm}^{2} / \mathrm{h}\right)$; Poids Organisque, P. cendres et poids Sec (mg/dm ${ }^{2}$.

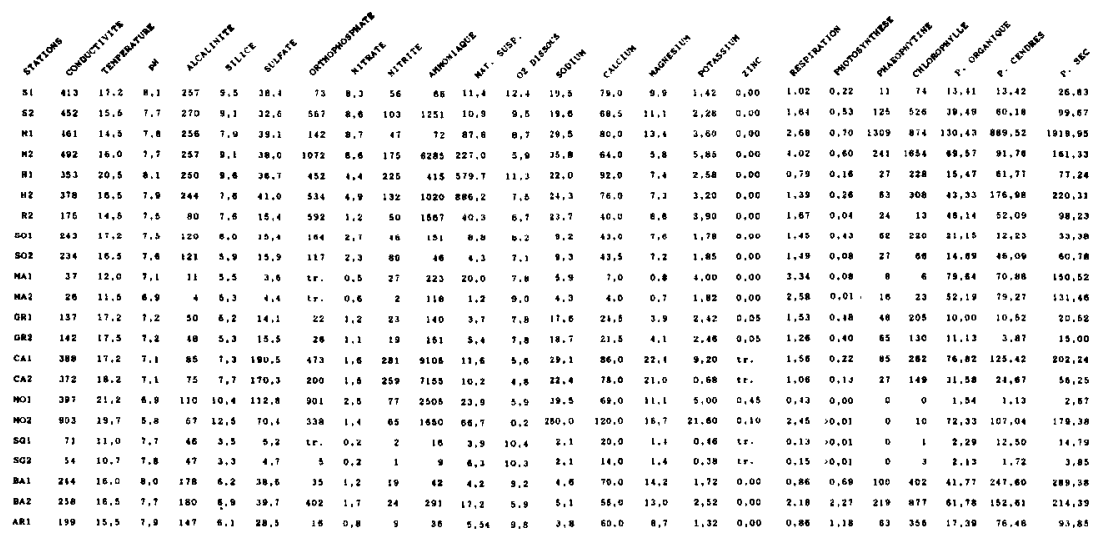

système des deux premiers axes (/ig. 10) permettent de dégager les points suivants :

(1) L'axe 1 range les stations depuis les caux faiblement chargées en éléments dissous et bien oxygénées (valeurs négatives), vers les eaux fortement chargées (valeurs positives). Aux premières correspondent des substrats peu chargés en périphyton, avec des activités photosynthétiques (TPr) et respiratoire (TRe) élevées. La figuration factorielle des relevés SG1 et SG2 caractérise ces stations comme les plus oligot rophes

Aux valeurs positives correspondent deux groupes de relevés qui se différencient par rapport à l'axe 2 :

Le premier groupe, associé aux forts développement de périphyton correspond aux stations les plus eutrophes (valeurs positives de l'axe 1 et 2). Le second groupe (valeurs négatives de l'axe 2) se caractérise au contraire par un faible développement des algues. Dans ce cas, une inhibition de la croissance des algues par la présence des toxiques est à considérer (teneurs en zinc aux stations MO1 et MO2, rejets d'industries chimiques en CA1 et CA2).

(2) L'influence des rejets polluants apparait au niveau de la position respective des stations situées à l'amont et à l'aval du point de rejet. L'effet de la pollution se traduit par un déplacement des relevés correspondants vers les valeurs positives de l'axe 1 (par exemple M1 - M2; S1 - S2; BA1 - BA2; MOI MO2). Cependant cette influence des rejets sur les rivières Salat (SG1 et SG2), Arnette (MA1 et MA2) et Dadou (GR1 et GR2) est moins évidente. Celà est vraisemblalement inhérent au fait que dans ces ruisseaux les stations amont (MA1, SG1, GRI) sont déjà influencees par des rejets polluants.

Une A.C.P. supplementaire (Watanabe 1985) considérant les espèces comme variables principales et les données de biomasse, d'activité et de physico-chimie de l'eau comme des paramètres supplémentaires a été effectuée afin de tester la valeur indicatrice des algues. Dans ce cas, les axes 1 et 2 expliquent seulement $32 \%$ de la variance. La figuration factorielle des stations identifiées par la 


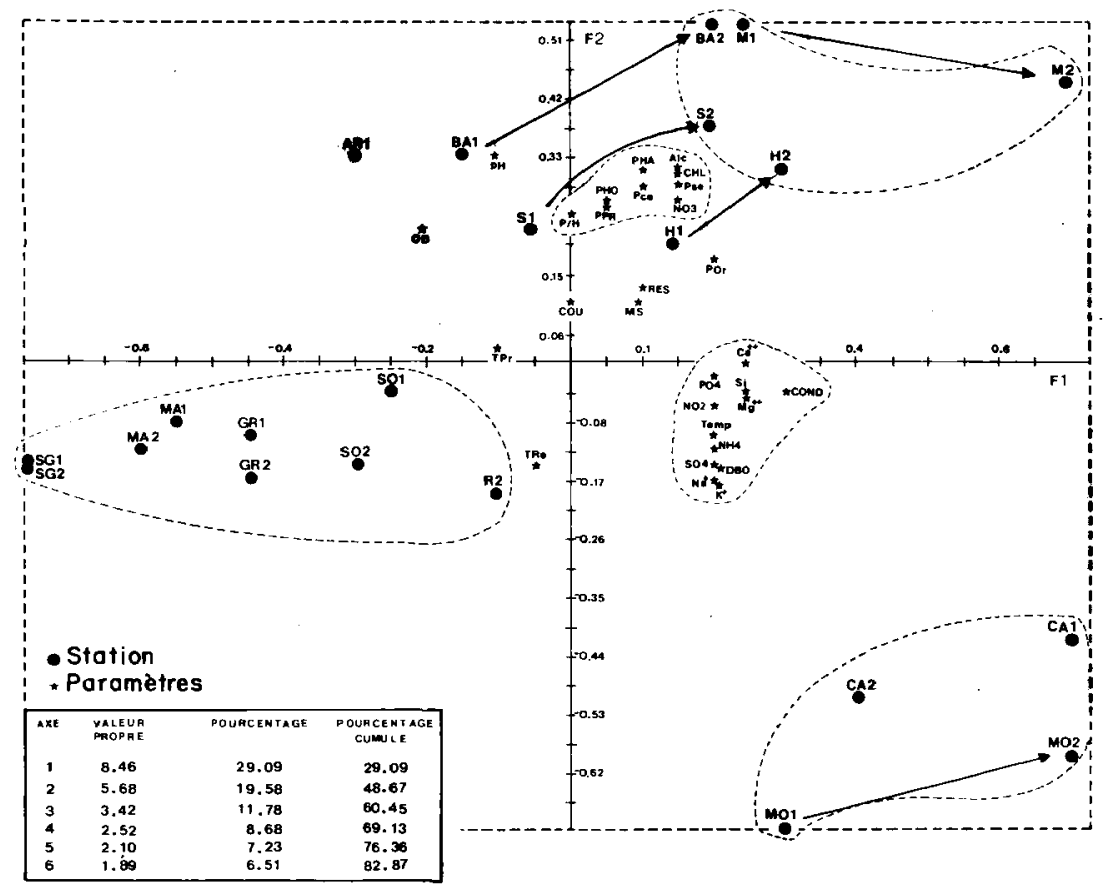

Fig. 10 : Analyse Factorielle en Composantes Principales. Projection des 22 relevés et des stations sur le plan factoriel des axes 1 et 2. PHA : phéophytines, PSe : poids sec, PCe : poids cendres, PHO = photosynthèse, PPR : production primaire, $\mathrm{P} / \mathrm{H}$ : autorophes/hétérotrophes, $\mathrm{POr}$ : poids organique, MS : matières en suspensions, COU : vitesse du courant, $\mathrm{TPr}$ : activité photosynthétique, TRe : activité respiratoire.

composition spécifique des communautés regroupe dans un même noyau d'affinité des eaux de caractéristiques physicochimiques très différentes. On peut en conlure que la structure du peuplement d'algues n'est pas le paramètre le plus sensible aux variations du niveau de pollution.

\section{Discussion}

Le périphyton est un microcosme dont le fonctionnement résulte de l'interact ion entre des processus autotrophes et hétérotrophes qui se déroulent dans la couche limite entre le subst rat solide et l'eau libre. Indépendamment des espèces qui la composent, cette communauté peut être caractérisée par des indices simples définissant globalement sa st ructure et sa productivité :

- chlorophylle et matière organique, témoins de l'importance relative des communautés autotrophes et hétérotrophes,

- taux d'accroissement de la biomasse 
- activité mélabolique (photosynthèse, respiration, activité hétérotrophe).

Afin de definir les relations entre ces indices et la qualité de l'eau, il était nécessaire de préciser:

- les modalités de croissance sur des supports artificiels.

- l'évolution des paramètres représentatifs de la structure et de l'activité du bioderme fixé en fonction de la durée d'immersion des substrats.

Nos observations ont confirmé que l'activité des microorganismes (taux de croissance et de métabolisme) et la structure qui en dépend varient avec l'áge des communautés fixées sur les substrats. Le développement de ces communautés aboutit prógressivement à la formation d'une couche constituée d'organismes vivants, sénescents ou morts, de matières organiques et minerales d'origine exogene, englobés dans une mat rice de polysaccharides (Lock 1971). Du point de vue de la croissance et du métabolisme, cette évolution de la communauté entraîne

1) une réduction de la diffusion des éléments nutritifs à l'interface ;

2) un fonctionnement relativement autonome, les bactéries bénéficiant des composés organiques libérés par les algues (production extraccllulaire el lyse des cellules mortes) qui utilisent à leur tour les composés minéraux issus de la dégradation bactérienne.

Il en résulte que le périphyton est plus directement lié aux conditions chimiques du milieu ambiant pendant la phase exponentielle de croissance que lorsque les communautés atteignent l'état d'équilibre (Capblancq \& Cassan 1979 b, SandJensen 1983).

La phase initiale de croissance fournit une bonne estimation de la productivité du périphyton (Kevern et al. 1966). Les bactéries maintiennent des taux de croissance élevés tant que l'épaisscur du bioderme reste relativement faible ot sa structure biologique assez simple, les pertes par broutage et par détachement étant alors peu importantes. Les supports vierges sont colonisés initialement par les bactéries dont le développement est lié aux tencurs en matières organiques de l'eau. Ce développement bactérien ent raine la formation d'une couche de polysaccharides qui constitue un prérequis a la fixation des microorganismes (Baier 1972). L'importance des bactéries comme organismes pionniers pendant la colonisation de substrats a été mise en évidence par des observations au microscope ćlectronique a balayage (Hudon \& Bourget 1981, Hoagland et al. 19 2). Néanmoins, selon Sieburth \& Thomas (1973), Paul et al. (1977), cet te colonisation primaire par des bactéries n'est pas indispensable aux Diatomées pour leur implantation.

La capacité d'échange entre les organismes et le milicu diminue avec l'épaississement du bioderme : ce phénomene qui correspond a la notion de couches limites évoquées par Sanders (1966) ríduit considérablement la diffusion des substrats organiques de l'eau vers les organismes. Les expériences de Wührmann (1969) mont rent qu'une augmentation de la turbulence accroit l'activité métabolique en favorisant cette diffusion.

L'utilisation des substrats artificicls doit surtout étre envisagée pour les études comparatives entre cours d'eau. La diversité des conditions physicochi. miques des écosystèmes d'eau courante est en effet telle qu'il apparaît difficile d'indiquer des plage's de valeurs de référence caractéristiques de l'état trophique ou du niveau de pollution d'un milieu.

L'avantage des substrats artificiels réside dans la possibilité offerte d'uniformiser les conditions de développement vis-à-vis des facteurs physiques (lumière, turbulence): La réponse des communautés périphytiques pendant la phase exponentielle de croissance est, dans ce cas, étroitement liée aux composantes chimiques du milieu aquatique. Nos résultats indiquent que dans ces conditions d'utilisation, les indices de structure et de productivité du périphyton constituent de bons indicateurs du niveau de pollution. Par rapport à ces indices, l'analyse de la structure spécifique des peuplements d'algues ne parait pas apporter d'informations complementaires.

\section{Travaux cités}

Baier (R.E.). 1982. - Influence of the initial surlace condition of materials on bioadhesion. Proc. Int. Cont. Mar. Corrosion Foul., 3: 633-639.

Capblancq (J.) \& Cassan (M.). 1979a. - Etude du periphyton d'unk rivière polluée (l'Agout). L. - Structure et développement des communautés sur substrats artificiels. Annls Limnol., 15 (2): 193-210.

Capblancq (J.) \& Cassan (M.). 1979b. - Etude du périphyton d'une rivière polluće (l'Agout). II. - Métabolisme et dynamique de croissance sur substrats artificiels. Annls Limnol, 15 (2): $211-222$.

Coste (M.). 1978. - Sur l'utilisation des Diatomées benthiques pour l'appréciation de la qualité biologique des eaux courantes: méthodologie comparée et approche typologique. Thèsc Doct. Sciences, Univ. Franche Comté, 143 p 
Descy (J.P.). 1976. - Etude quantitative du peuplement algal benthique en vue de l'établissement d'une méthodologie d'estimation biologique de la qualité des eoux courantes: application au cours belge de la Meuse et de la Sambre. In : Recherche et Technique au service de l'environnement, Cebedoc (Ed.), Liège : 159-206.

Descy (J.P.). 1979. - A new apprcach to water quality estimation using diatoms. Nova Hedw., 64 : 305-323.

Fichenberger (E.). 1975. - On the quantitative effects of chemical factors on running water ecosystems. Schweiz. Z. Hydrol, $37: 21-34$.

Herbland (A.M.) \& Bois (J.F.). 1974. - Assimilation et minéralisation de la matière organique dissoute dans la mer : méthode par le comptage en scintillation liquide. Marine Biology, 24 : 203-212.

Hoagland (K.D.). Roemer (S.C.) \& Rosowski (J.R.). 1982. Colonisation and community structure of two periphytic assemblages, with emphasis on the diatoms (Bacillariophyceae). Amer. J. Bot., $69:$ 188-213.

Hubbie (J.E.) \& Wright (R.T.). 1965. - Competition between plank. tonic bacteria and algae for organic solutes. Memorie Ist. hal. Idrobiol., Suppl. $18 ; 175-185$.

Hudon (C.) \& Bourget (E.). 1981. - Initial colonization of artificial substrate ; community development and structure studied by scanning electron microscopy. Can. J. Fish. Aquat. Sci., 38 : 1371.1384

Kevern (N.R.), Wilhm (J.L.) \& Van Dyne (G.M.). 1966. - The use of artificial substrata to estimate the productivity of periphy. ton. Limnol. Oceanogr., 11 : 499-502.

lange-Berthalot $\left(\mathrm{H}_{1}\right)$. 1979. - Pollution tolerance of diatoms as a criterion for water quality estimation. Nova Hedw., 64 : 285-304.

Lock (M.A.). 1981. - River epilithon. A light and organic energy transducer. In Lock M.A. \& Williams D.D. : Perspective in running water ecology. Plenum Press, N.Y. : 3-40.
Paul (R.W. Jri, Kuhn (D.L.), Plafkin (J.L.), Cairns (J. Jr) \& Croxdale (J.G.). 1977. - Evaluation of natural and artificial substrate colonization by scanning electron microscopy. Trans. Amer. Microsc. Soc, 96 : 506-519.

Sanders (W.M.). 1966. - Oxygen utilization by slime organisms in continuous culture, Air and Wat. Pollut. Int. J., 10 (4) : 253-276.

Sand-Jensen (K.). 1983. - Physical and chemical parameters regulating growth of periphytic communities. In Wetzel R.G.: Periphyon of freshwater ecosystems. W. Junk Publishers. The Hague : 63-71.

Sieburth (J. McN.) \& Thomas (C.D.). 1973. - Fouling on eelgrass (Zoostera marina L.). J. Phycol., 9 ; 46-50.

Sullivan (M.J.). 1986. - Mathematical expression of diatoms results : are these "pollution indices * valid and useful ?. In 8th Diatom Symposium Proceedings, 1984, Paris, Ricard M. (Ed.), S. Koeltz, Koenigstein : 772-776.

Watanabe (T.) 1983. - Etude de la relation entre le périphyton et la qualite chimique de l'eau des rivieres : utilisation de bioessais * in situ * (substrats artificiels) pour caractériser l'état de pollution des eaux. These Université, Toulouse III, $120 \mathrm{p}$.

Wetzel (R.G.). 1983. - Periphyton of freshwater ecosystems. Development in Hydrobiology, $\mathrm{N}^{\circ} 17, \mathrm{~W}$. Junk Publishers, The Hague : $346 \mathrm{p}$.

Wührmann (K.). 1969. - Selbst reinigung in Fließgewässern. Europ. Abwassersymposium, Mïnchen, A.T.V., $23: 15-32$.

Wührmann (K.). 1974. - Some problems and perspectives in applied limnology. Mitt. Intemat. Verein. Limnol., 19 : 2028-2034.

Wührmann (K.) \& Eichenberger (E.). 1975. - Experiments on the effects of inorganic enrichment of rivers on periphyton primary production. Verh. Internat. Verein. Limnol., 19 : 2028.2034. 